\title{
SpS1-Laboratory spectroscopy of small molecules
}

\author{
Peter F. Bernath \\ Department of Chemistry, University of York, Heslington, York, YO10 5DD, UK \\ email: pfb500@york.ac.uk
}

This contribution focuses on the study of 'cool' sources with surface temperatures in the range of about $500-4000 \mathrm{~K}$. In this temperature range spectra are dominated by strong molecular absorption and the tools of modern chemical physics can be applied to compute the molecular opacities needed to simulate the observed spectral energy distributions. (See Bernath (2005) for an introduction to molecular spectroscopy including line intensities and Bernath (2009) for a recent astronomical review article.)

\section{Hot $\mathrm{H}_{2} \mathrm{O}, \mathrm{CH}_{4}$ and $\mathrm{NH}_{3}$}

The vibration-rotation and pure rotational lines of $\mathrm{H}_{2} \mathrm{O}$ and $\mathrm{OH}$ appear strongly in cool oxygen-rich objects. The dominant carbon-containing molecule is $\mathrm{CO}$ at high temperatures and $\mathrm{CH}_{4}$ for cooler objects, such as T-type brown dwarfs and 'hot Jupiter' extrasolar planets. For nitrogen, $\mathrm{N}_{2}$ is the high temperature molecular form, but $\mathrm{NH}_{3}$ appears in $\mathrm{T}$ dwarfs and is predicted to be the distinguishing feature for very cool brown dwarfs ('Y'-type) with surface temperatures below about $700 \mathrm{~K}$.

Excellent high resolution ground-based spectra of the Sun are available from the Kitt Peak Fourier transform spectrometer (FTS) and from low earth orbit with the Advanced Composition Explorer (ACE) FTS. The spectra are dominated by the strong first overtone and fundamental vibration-rotation bands of $\mathrm{CO}$. The Meinel bands ( $\mathrm{OH}$ vibration-rotation bands) near $3 \mu \mathrm{m}$ are also strong and the $\mathrm{OH}$ pure rotational lines are prominent at longer wavelengths. The vibration-rotation bands of $\mathrm{CH}$ and $\mathrm{NH}$ can be seen at $3 \mu \mathrm{m}$ and pure rotational lines of $\mathrm{NH}$ are also present.

A new very high signal-to-noise solar atlas in the $750-4400 \mathrm{~cm}^{-1}$ region has recently been prepared by Hase et al. (2009) from ACE satellite observations. Based on ACE, ATMOS and laboratory spectra, the analysis of $\mathrm{OH}$ has recently been improved by Bernath \& Colin (2009) and similar work is underway for $\mathrm{CH}$ and $\mathrm{NH}$.

At $5800 \mathrm{~K}$, the Sun's photosphere is too hot for water to exist, but by $3900 \mathrm{~K}$ the concentration of $\mathrm{OH}$ and $\mathrm{H}_{2} \mathrm{O}$ are equal. A large number of unassigned lines were noticed in two Kitt Peak sunspot atlases; it was suspected that these lines were due to hot water but the available laboratory data were inadequate to confirm this. Comparison of a new laboratory emission spectrum of $\mathrm{H}_{2} \mathrm{O}$ at $1800 \mathrm{~K}$ with the sunspot absorption spectrum identified most of the unassigned sunspot lines as $\mathrm{H}_{2} \mathrm{O}$ lines. Wallace et al. (1995) proved that there is 'water on the Sun'. Through variational calculations of the energy levels using a high quality ab initio potential energy surface, Polyansky et al. (1997) were able to assign most of the strong lines.

The water emission in the $500-13000 \mathrm{~cm}^{-1}$ spectral region was recorded with a high-resolution FTS using an oxy-acetylene torch as a source. Work on this $3000 \mathrm{~K}$ spectrum of $\mathrm{H}_{2} \mathrm{O}$ has just finished with the publication by Zobov et al. (2008) of the last paper in the series. The latest water linelist of Barber et al. (2006) contains more than 500 million lines and is recommended for simulation of spectral energy distributions of cool stars, brown dwarfs and extrasolar planets.

The situation for $\mathrm{CH}_{4}$ is much less satisfactory than for $\mathrm{H}_{2} \mathrm{O}$ although Nassar \& Bernath (2003) have published hot emission spectra and there is considerable theoretical activity, for example by Warbier et al. (2009) A similar situation exists for $\mathrm{NH}_{3}$ with calculations underway, for instance, by Huang et al. (2008)

We have decided to take an empirical approach to determine new linelists for hot $\mathrm{CH}_{4}$ and $\mathrm{NH}_{3}$. A reasonably complete list of line positions, line strengths and lower state energy levels is needed. We have therefore begun to record spectra of hot $\mathrm{NH}_{3}$ and $\mathrm{CH}_{4}$ over a wide range of temperatures and to calibrate the line positions and strengths using the HITRAN database 
of Rothman et al. (2009) Empirical lower state energies are determined from the temperature dependence of the line intensities.

\section{Metal hydrides}

The only metal hydride seen clearly in the solar photosphere spectrum is $\mathrm{MgH}$, detected via the $\mathrm{A}^{2} \Pi-\mathrm{X}^{2} \Sigma^{+}$transition. Sunspot spectra convincingly show lines of the visible and near infrared electronic transitions of $\mathrm{AlH}, \mathrm{MgH}, \mathrm{CaH}$ and $\mathrm{FeH}$. Both the $\mathrm{A}^{2} \Pi-\mathrm{X}^{2} \Sigma^{+}$and $\mathrm{B}^{\prime 2} \Sigma^{+}-$ $\mathrm{X}^{2} \Sigma^{+}$electronic transitions of $\mathrm{MgH}$ can be seen in sunspot spectra. Shayesteh et al. (2003) completed a set of $\mathrm{MgH}$ observations in the infrared and visible that had led earlier in 2003 to the discovery of the linear $\mathrm{MgH}_{2}$ molecule. From the $\mathrm{MgH} \mathrm{B}-\mathrm{X}$ observations, Shayesteh et al. (2007) determined all bound ground state levels with spectroscopic precision and obtained a very precise dissociation energy of $11104.7 \mathrm{~cm}^{-1}$.

The most important application of the spectroscopy of metal hydrides is in the classification of L-type dwarfs as discussed by Kirkpatrick (2005). The FeH F ${ }^{4} \Delta-\mathrm{X}^{4} \Delta$ electronic transition near $1 \mu \mathrm{m}$ and the $\mathrm{E}^{4} \Pi-\mathrm{A}^{4} \Pi$ transition near $1.58 \mu \mathrm{m}$ are seen strongly along with the $\mathrm{A}^{6} \Sigma^{+}-\mathrm{X}^{6} \Sigma^{+}$ transition of $\mathrm{CrH}$ near $861 \mathrm{~nm}$. Dulick et al. (2003) prepared a linelist for the $\mathrm{F}-\mathrm{X}$ transition of $\mathrm{FeH}$, Burrows et al. (2002) prepared one for $\mathrm{CrH}$ and Burrows et al. (2005) for $\mathrm{TiH}$ (which has not been detected yet in L-dwarfs) by extrapolating laboratory observations and using line intensities based on ab initio calculations. These molecular opacities are in modest agreement with observations, but high resolution comparisons indicate that improvements are needed.

\section{References}

Barber, R. J., Tennyson, J., Harris, G. J. \& Tolchenov 2006, MNRAS 368, 1087

Bernath, P. F. 2005, Spectra of Atoms and Molecules, Second Edition, Oxford UP, New York

Bernath, P. F. 2009, Int. Rev. Phys. Chem., in press

Bernath, P. F. \& Colin, R. 2009, J. Mol. Spectrosc., in press

Burrows, A., Ram, R. S., Bernath, P., Sharp, C. M. \& Milsom, J. A. 2002, Astrophys. J. 577, 986

Burrows, A., et al. 2005, ApJ 624, 988

Dulick, M., et al. 2003, ApJ 594, 651

Hase, F., Wallace, L., McLeod, S. D., Harrison, J. J. \& Bernath, P. F. 2009, J. Quant. Spectrosc. Rad. Transfer, in preparation

Huang, X., Schwenke, D. W., \& Lee, T. J. 2008, J. Chem. Phys. 129, 214304

Kirkpatrick, J. D. 2005, Annu. Rev. Astron. Astrophys. 43, 195

Nassar, R. \& Bernath, P. 2003, J. Quant. Spectrosc. Rad. Transfer, 82, 279

Polyansky, O. L., et al. 1997, Science 277, 346

Rothman, L. S., et al. 2009, J. Quant. Spectrosc. Rad. Transfer 110, 533

Shayesteh, A., et al. 2007, J. Phys. Chem. A 111, 12495

Shayesteh, A., Appadoo, D. R. T., Gordon, I. \& Bernath, P. F. 2003, J. Chem. Phys. 119, 7785

Wallace, L., et al. 1995, Science 268, 1155

Warmbier, R., et al. 2009, A\&A 495, 655

Zobov, N. F., et al. 2008, MNRAS 387, 1093 